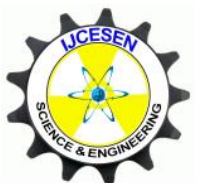

Copyright (C) IJCESEN
International Journal of Computational and

Experimental $\boldsymbol{S}$ cience and $\boldsymbol{E N}$ ineering

(IJCESEN)

Vol. 6-No.3 (2020) pp. 145-151

http://dergipark.org.tr/en/pub/ijcesen

Research Article

\title{
Factors Affecting “Organs at Risk" Doses in 3 Dimensional Brachytherapy (3D BRT) of Cervical and Endometrium Cancers
}

\author{
Nazmiye Deniz ARSLAN ${ }^{1 *}$, Hazım Orhan KIZILKAYA ${ }^{2}$, Ayşe KUTLUHAN DOĞAN ${ }^{1}$ \\ ${ }^{1}$ Health Sciences Üniversity, Yedikule Chest Diseases And Chest Surgery Training And Research Hospital, Radiation \\ Oncology, 34020, İstanbul-Turkey \\ ${ }^{2}$ Health Sciences Üniversity, Şişli Hamidiye Etfal Training And Research Hospital, Radiation Oncology, 34360, \\ İstanbul-Turkey
}

* Corresponding Author: denizsaracoglu@ hotmail.com ORCID: 0000-0003-0080-2284

\section{Article Info:}

DOI: $10.22399 /$ ijcesen.799808

Received : 29 September 2020

Accepted : 26 October 2020

\section{Keywords:}

Brachythreapy

volume of bladder

cervix cancer

endometrial cancer

\begin{abstract}
$\underline{\text { Abstract: }}$
In brachytherapy, both the tumor and organ at risk may have intrafractional and interfractional anatomical differences. In this study, we aimed to investigate the factors affecting Organs at Risk (OAR) doses in adaptive 3D BRT applications. A total of 55 patients that underwent intracavitary brachytherapy with the diagnosis of a gynecological tumor between September 2012 and December 2013 were evaluated retrospectively. The effect of the surgery, applicator type, bladder, rectum and sigmoid on $\mathrm{D}_{2 \mathrm{cc}}, \mathrm{D}_{1 \mathrm{cc}}, \mathrm{D}_{0.1 \mathrm{cc}}$ values were investigated. The median age was 55 years. While there was no statistically significant difference between $1^{\text {st }}$ and $2^{\text {nd }}$ fractions measurements, the sigmoid $D_{1 c c}$ values between the $1^{\text {st }}$ and $3^{\text {rd }}$ fractions were found to be statistically significant $(p=0.004)$. When the effect of the interfractional change of bladder filling on OAR doses in the first three fractions was examined, it was not statistically significant however, its effect on bladder, sigmoid and rectum $D_{2 c c}, D_{1 c c}, D_{0.1 c c}$ values were observed in some fractions. The bladder point dose was found to be statistically significant in operated patients $(\mathrm{p}=$ 0.005). When the comparison was made according to the application type, the bladder dose was found to be statistically significant in roller applications than the other two applicator treatments $(\mathrm{p}=0.001)$. 3D BRT provides maximum protection of healthy tissues while giving a high dose to the target as a result of adaptive planning by performing computed tomography in each fraction. The sigmoid is the OAR that makes the most distinctive interfraction. To obtain higher treatment accuracy in each fraction, routine preparation and tomographic imaging followed by adaptive planning should be done.
\end{abstract}

\section{Introduction and Purpose}

According to GLOBOCAN 2020 data, amongst gynecologic cancers, endometrial cancers are the most common in developed countries and cervical cancers are the most common. Following these, endometrial cancers are the second most common cancer type [1]. In our country, endometrium cancer is the most common. Although the primary treatment is surgery in endometrial cancers, Intracavitary Brachytherapy is also one of the most important complementary steps of treatment, especially in the early stages. In cervical cancers, earlystage surgery is at the forefront however, radiotherapy is the first option in advanced stage [1, 2, 3] cancers.
Intracavitary Brachytherapy, alone or together with External Radiotherapy, increases local control in both cancers $[2,4,5]$. Bladder, sigmoid and rectum toxicity rates, which are known as organs at risk in these patients, are proportional to the total doses received from External RT \pm Brachytherapy. Chronic cystitis, fibrosis, and vesicovaginal fistulas might occur in the bladder, and rectitis and fistulas might occur in the rectum. It was reported that with radiotherapy, mild bladder complications were between $8-12 \%$ and serious bladder complications were between 2-6\% [3]. Knowing the factors that are effective in Organs at Risk (OAR) doses allows us to predict the risk of complications, and make necessary changes in planning. In many centers, 3D 
High-Dose Rapid (3D HDR) Brachytherapy is applied by using Computed Tomography (CT) in the first fraction (fr) however, treatment is applied in following fractions with the same plan as conventional graphics are obtained with a C-arm. In this practice, the effect of interfractional volume changes on target treatment volumes cannot be observed. Target volumes can be better determined with adaptive brachytherapy and the appropriate dose may thus be given, reducing OAR doses. Tumor regression and OAR movements might change the applicator placement and angle. In this case, Adaptive Intracavitary Brachytherapy is recommended with imaging in each application to see the shrinkage of the tumor and determine the exact doses of OAR $[4,5]$. It is possible to increase local control with an Adaptive 3D BRT, while reducing the side effects stemming from OAR doses. In the present study, clinical factors that might be effective in OAR doses in Adaptive 3D BRT applications were investigated, and the effect of these factors on dose-volume curves was examined statistically.

\section{Materials \& Method}

Patients who were diagnosed with gynecological tumors and who underwent Intracavitary Brachytherapy between September 2012 and December 2013 were evaluated retrospectively. A total of 55 patients diagnosed with endometrium cancer, and 32 with cervical cancer were included in the study. The ages of the patients ranged between 26 and 84, and the median age was 55. The pathological diagnosis of the cervical cancer patients were squamous-cell carcinoma in 27 patients, adenoid in 4 patients, and adenoid-squamous carcinoma in 1 patient. The pathological diagnosis of the endometrial cancer patients were adenocarcinoma in 22 patients, and serous papillary carcinoma in 1 patient. A total of 21 patients with endometrium cancer underwent $\mathrm{TAH}+\mathrm{BSO}+\mathrm{PELVIC} \mathrm{LND} \pm \mathrm{PA}$ LND (2 patients were inoperable), and 9 patients with cervical cancer were operated on. Two patients with endometrial cancer were medically inoperable, 6 patients were Stage 2, 7 patients were Stage 3, and 8 patients were stage 1 . A total of 9 patients with cervical cancer were Stage 1B2; and among the inoperable patients, 15 were Stage $2 \mathrm{~B}, 1$ was Stage $3 \mathrm{~A}$, and 7 were Stage 3B. All patients diagnosed with cervical cancer underwent External Pelvic RT (50.4Gy) \pm Simultaneous Chemotherapy $\left(40 \mathrm{mg} / \mathrm{m}^{2} /\right.$ weekly cisplatin), and only 13 of the endometrial cancer patients underwent External Pelvic RT (45-50.4Gy).

A BRT (Nucletron, Vendaal, Netherlands) was applied to the patients by using Linear Accelerator (Trilogy ${ }^{\mathrm{TM}}$; Varian Medical Systems, Palo Alto, CA, USA- Oncor ${ }^{\mathrm{TM}}$; Siemens AG, Munich, Germany) radiotherapy devices and post-Pelvic EBRT MR/CT compatible gynecological applicators. In Postop BRT applications, the upper 1/3 of the vagina was determined as the target area by using cylinder applicators suitable for the patient's vagina size. In 30 patients with postoperative gynecological tumors (21 endometrium ca +9 cervix ca), the cylinder applicator was used, and in patients with inoperable gynecological tumors, the Adaptive 3D HDR BRT was performed by using 22 tandem + ring applicators (22 cervix ca) and 3 cylinder + tandem applicator (2 endometrium ca +1 cervix ca). The effects of operation presence, applicator type, OAR volumes on the $\mathrm{D}_{2} \mathrm{cc}$, $\mathrm{D}_{1 \mathrm{cc}}, \mathrm{D}_{0.1 \mathrm{cc}}$ values of the bladder, rectum and sigmoid were investigated.

\section{Brachytherapy}

The patients were informed about the application before the treatment. All abdominal MRI and gynecological examinations of each patient were performed before the BRT. In the examinations, size of the vagina, size and position of the uterus, localization, dimensions and diffusion of tumors, and the type of applicators to be used were determined. All patients were given prebrachytherapy bowel cleansing training (Enema Application in the $12^{\text {th }}$ hour and $3^{\text {rd }}$ hour before the treatment). The patients were rested in the gynecological examination position at the special treatment desk. The balloon was inflated by administering a bladder probe (2-way Foley probe, Beybi ${ }^{\circledR}$, Turkey) and 7 cc Radioopaque Substance (Omnipaque $350 \mathrm{mg} / \mathrm{mL}$; GE Healthcare, Little Chalfont, UK) to the patients. Then, CT/MR compliant applicators were placed and fixed, and the planning CTs were performed by administering 20 cc contrast Normal Saline (NS) to the bladder. Cylinder applicators were used for postop patients, a Ring + Tandem Applicator was used for inoperable patients, and a Cylinder + Tandem Applicators were used for only 3 patients. The endometrium lumen lengths of the patients were measured with a Hysterometer, and the appropriate tandem length was determined (min 2$\max 6 \mathrm{~cm}$ ). In postop patients, the appropriate cylinder diameter was determined according to the size of the vagina that was measured during vaginal examination (min 2-max $3.5 \mathrm{~cm})$.

\section{Planning}

In each fraction, Pelvic CTs (Toshiba Alexion, 16-slice, Japan) were performed with $3 \mathrm{~mm}$ sections in the supine position approximately 15 minutes after the applicator in the brachytherapy unit (by applying general anesthesia when necessary). The CT images were transferred to the Oncentra Treatment Planning System (TPS) (Oncentra version 4.5.3.30, Nucletron, Vendaal, Netherlands), and High-Risk Clinical Target Volume (HR CTV) and OAR (bladder, rectum and sigmoid) volumes were contoured for patients with cervical cancer. The CTV (vaginal cuff), PTV (CTV+0.5mm margin) and OAR volumes were contoured for patients with endometrium cancer in line with the rules specified in the GEC ESTOAR and ICRU 38 report. The HR CTV, PTV, bladder $\mathrm{D}_{2 \mathrm{cc}}$ (the dose taken as maximum $2 \mathrm{~cm} 3$ of the tissue volume), $\mathrm{D}_{1 \mathrm{cc}}, \mathrm{D}_{0.1 \mathrm{cc}}$ values, rectum $\mathrm{D}_{2 \text { cc }}, \mathrm{D}_{1 \mathrm{cc}}, \mathrm{D}_{0.1 \mathrm{cc}}$ values, and sigmoid $\mathrm{D}_{2 \mathrm{cc}}, \mathrm{D}_{1 \mathrm{cc}}, \mathrm{D}_{0.1 \mathrm{cc}}$ values were calculated, and the bladder fullness was recorded in cc.

\section{Treatment}

According to the recommendations of the American Brachytherapy Society (ABS), the number and dose of the fractions were determined based on the condition of external radiotherapy and operation status of the patients. 
After the treatment doses and the appropriate treatment plans were determined, the patients were treated with Ir192 after loading HDR BRT (Nucletron, Vendaal, Netherlands) (10 Ci source activities) twice a week. In postop patients, the 5-mm depth of the vaginal mucosa was determined as the PTV target tissue. The distribution of bladder volumes were examined, and bladder fullness values were divided into 3 groups as $<90 \mathrm{cc}, 90-110 \mathrm{cc}$, and >110 cc. For HR-CTV, D90 (HRCTV's minimum dose taken by \%90) and for organs at

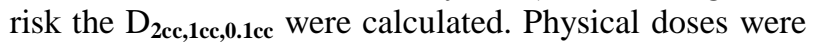
converted to the EQD2 Linear Quadratic Model that was $\mathrm{a} / \beta=10$ Gy for HR-CTV, and $\mathrm{A} / \beta=3$ Gy for OARs. The planning target was EQD2 $\mathrm{D}_{90} \geq 85$ Gy (total dose EBRT + BT) for HR-CTV. For OARs, the $\mathrm{D}_{2 \mathrm{cc}}$ values were EQD2 90 Gy for the bladder, and EQD2 75 Gy for the rectum and sigmoid. The Fr count and dose of the patients were calculated by taking into account these values.

\section{Statistics}

The SPSS (Version 17.0; SPSS Inc., Chicago, IL) program was used in the statistical analysis of the data. The categorical measurements were summarized as numbers and percentages, and continuous measurements were summarized as mean and standard deviation (median and min-max when needed). The dosimetric analyses were made separately for each application method and doses were compared among the three application methods. For the evaluation of the data according to the application types, the Kruskal Wallis Test and Mann Whitney U-test were used (cylinder and tandem ring comparison), and the Mann Whitney U-test was used to analyze the effect of the operation presence. The difference between the basal values and other measurements was evaluated with the Wilcoxson Test. The statistical significance level was taken as $p=0.05$ in all tests.

N.B.: This study was presented in March 2014 as a dissertation under the same name.

\section{Results}

The median age was 55 years (min:26 - max:84). A total of 23 of the patients had endometrium cancer (22 adenoma, 1 serous papillary ca) and 32 had cervix cancer (27 squamous hc, 4 adenoma, 1 adenosquamous ca). The bladder, sigmoid and rectum $\mathrm{D}_{2 \mathrm{cc}}, \mathrm{D}_{1 \mathrm{cc}}, \mathrm{D}_{0.1 \mathrm{cc}}$ values had interfractional changes, in other words, the measurements were in basal values. When the $2^{\text {nd }}$ and $3^{\text {rd }}$ fractions of the 55 patients who were diagnosed with cervical and endometrium cancer were compared, significant differences were detected only in sigmoid $1^{\text {st }}$ and $3^{\text {rd }}$ fr $D_{2}$ cc $, D_{1 c c}, D_{0.1 c c}$. Although there were no statistically significant differences between the $1^{\text {st }}$ fr and $2^{\text {nd }}$ fr measurements, the Sigmoid $D_{1 \mathrm{cc}}, D_{1 \mathrm{cc}}$ values between the $1^{\text {st }}$ and $3^{\text {rd }}$ fractions were found to be statistically significant $(p=0.004)$. Although Sigmoid 1.frc $\mathrm{D}_{1 \mathrm{cc}}$ was $482.2 \mathrm{cGy}(122.5-946 \mathrm{cGy})$, Sigmoid 3.frc was found to be $\mathrm{D}_{1 \mathrm{cc}} 395 \mathrm{cGy}(82.0-812.5 \mathrm{cGy})$. An interfractional decrease was found to be statistically significant(Table 1).

Table 1: Comparison of basal and other measurement values

\begin{tabular}{|c|c|}
\hline $\begin{array}{l}\text { OAR } D_{1 \mathrm{cc}} \text { and } \mathrm{D}_{0.1 \mathrm{cc}} \text { 1.fre and 2. and 3.frc } \\
\text { values }\end{array}$ & $\mathbf{p}$ \\
\hline rectum $1 \mathrm{cc} 1 \mathrm{frc}-\mathrm{r} 1 \mathrm{cc} 2 \mathrm{frc}$ & 0.319 \\
\hline rectum $1 \mathrm{cc} 1 \mathrm{frc}-\mathrm{r} 1 \mathrm{cc} 3 \mathrm{frc}$ & 0.346 \\
\hline bladder1cc $1 \mathrm{frc}-\mathrm{m} 1 \mathrm{cc} 2 \mathrm{frc}$ & 0.645 \\
\hline bladder1cc 1 frc - m1cc 3frc & 0.332 \\
\hline sigmoid 1cc1frc - s1cc 2 frc & 0.573 \\
\hline sigmoid 1cc1frc - s1cc 3frc & 0.004 \\
\hline rektum0.1cc1frc - r0.1cc $2 \mathrm{frc}$ & 0.140 \\
\hline rektum0.1cc1frc - r0.1cc 3frc & 0.080 \\
\hline bladder0.1cc1frc - m0.1 cc2frc & 0.538 \\
\hline bladder0.1cc1frc - m0.1 cc3frc & 0.465 \\
\hline sigmoid0.1cc1frc - s0.1cc 2 frc & 0.101 \\
\hline sigmoid0.1cc1frc - s0.1cc 3frc & 0.002 \\
\hline
\end{tabular}

$\mathrm{P}=<0,05$

29 of the 55 patients were treated with 3 frc, 26 were treated with 4 frc. It was aimed that the frc count was increased to 4 and the dose of the OAR per fraction was reduced for 23 inoperable patients with cervical cancer, 2 inoperable endometrium cancer patients, and 1 operable endometrium cancer patient to optimize the OAR tolerance doses. The patients were divided into 3 groups according to bladder volumes as $<90 \mathrm{cc}, 90-110$ cc, and >110 cc (Table 2).

Table 2: Distribution of Bladder Fullness Rate according to Bladder Volumes

\begin{tabular}{|c|c|c|}
\hline & Number & $\%$ Rate \\
\hline \multicolumn{3}{|l|}{$\begin{array}{l}\text { Bladder full. } \\
\text { 1.frc }\end{array}$} \\
\hline$\leq 90$ & 15 & 27.3 \\
\hline $90-110$ & 20 & 36.4 \\
\hline$>110$ & 20 & 36.4 \\
\hline \multicolumn{3}{|c|}{\begin{tabular}{l|l} 
Bladder full. & \\
2.frc &
\end{tabular}} \\
\hline$\leq 90$ & 17 & 30.9 \\
\hline $90-110$ & 16 & 29.1 \\
\hline$>110$ & 22 & 40.0 \\
\hline \multicolumn{3}{|l|}{$\begin{array}{l}\text { Bladder full. } \\
\text { 3frc }\end{array}$} \\
\hline$\leq 90$ & 11 & 20.0 \\
\hline $90-110$ & 17 & 30.9 \\
\hline$>110$ & 27 & 49.1 \\
\hline \multicolumn{3}{|l|}{$\begin{array}{l}\text { Bladder full. } \\
\text { 4frc }\end{array}$} \\
\hline$\leq 90$ & 3 & 11.5 \\
\hline $90-110$ & 9 & 34.6 \\
\hline$>110$ & 14 & 53.8 \\
\hline
\end{tabular}

When the effect of interfractional change of bladder fullness on OAR doses in the first 3 fractions were 
examined, there were effects on the bladder, sigmoid and rectum $\mathrm{D}_{2 \mathrm{cc}}, \mathrm{D}_{1 \mathrm{cc}}, \mathrm{D}_{0.1 \mathrm{cc}}$; however, these effects were not at statistically significant levels (Table 3 ).

Table 3: Effect of Bladder Fullness on OAR Doses in all Fractions

\begin{tabular}{|c|c|c|c|c|c|c|c|}
\hline $\begin{array}{l}\text { Bladder } \\
\text { fullness }\end{array}$ & \multicolumn{2}{|c|}{$\leq 90$} & \multicolumn{2}{|c|}{$90-110$} & \multicolumn{2}{|c|}{$>110$} & \multirow[b]{2}{*}{$\mathrm{p}$} \\
\hline (cGy) & $\mathrm{n}$ & $\begin{array}{l}\text { Mean } \\
\text { (Min- } \\
\text { Max) }\end{array}$ & $\mathrm{n}$ & $\begin{array}{l}\text { Mean } \\
\text { (Min- } \\
\text { Max) }\end{array}$ & $\mathrm{n}$ & $\begin{array}{l}\text { Mean } \\
\text { (Min- } \\
\text { Max) }\end{array}$ & \\
\hline $\begin{array}{l}\text { rectum } 2 \mathrm{cc} \\
1 . \text { frc }\end{array}$ & $\begin{array}{l}1 \\
5\end{array}$ & $\begin{array}{l}361(1 \\
34- \\
555)\end{array}$ & 20 & $\begin{array}{l}384(72 \\
-519)\end{array}$ & 20 & $\begin{array}{l}383(2 \\
26- \\
562)\end{array}$ & $\begin{array}{l}0.5 \\
94\end{array}$ \\
\hline $\begin{array}{l}\text { mesane2cc } \\
1 . f r c\end{array}$ & $\begin{array}{l}1 \\
5\end{array}$ & \begin{tabular}{|l|}
$351(2$ \\
$46-$ \\
$593)$ \\
\end{tabular} & 20 & $\begin{array}{l}404(33 \\
1-602)\end{array}$ & 20 & $\begin{array}{l}447(3 \\
05- \\
681) \\
\end{array}$ & $\begin{array}{l}0.0 \\
26\end{array}$ \\
\hline $\begin{array}{l}\text { sigmoid } 2 \\
\text { cc1.frc }\end{array}$ & $\begin{array}{l}1 \\
5\end{array}$ & \begin{tabular}{|l|}
$279(9$ \\
$2-$ \\
$495)$ \\
\end{tabular} & 20 & $\begin{array}{l}264(10 \\
4-582)\end{array}$ & 20 & $\begin{array}{l}314(1 \\
56- \\
525) \\
\end{array}$ & $\begin{array}{l}0.4 \\
64\end{array}$ \\
\hline $\begin{array}{l}\text { rectum } \\
1 \mathrm{cc} 1 \mathrm{frc}\end{array}$ & $\begin{array}{l}1 \\
5\end{array}$ & $\begin{array}{l}403(1 \\
47- \\
602)\end{array}$ & 19 & $\begin{array}{l}412(29 \\
2-561)\end{array}$ & 19 & $\begin{array}{l}427(2 \\
47- \\
620)\end{array}$ & $\begin{array}{l}0.3 \\
98\end{array}$ \\
\hline $\begin{array}{l}\text { mesane1cc } \\
1 \text { frc }\end{array}$ & $\begin{array}{l}1 \\
5\end{array}$ & $\begin{array}{l}384(2 \\
73- \\
613)\end{array}$ & 19 & $\begin{array}{l}436(36 \\
0-654)\end{array}$ & 19 & $\begin{array}{l}468(3 \\
30- \\
743)\end{array}$ & $\begin{array}{l}0.0 \\
45\end{array}$ \\
\hline $\begin{array}{l}\text { sigmoid } \\
1 \mathrm{cc} 1 \mathrm{frc}\end{array}$ & $\begin{array}{l}1 \\
5\end{array}$ & $\begin{array}{l}269(9 \\
7- \\
625) \\
\end{array}$ & 20 & $\begin{array}{l}405(11 \\
5-648)\end{array}$ & 20 & $\begin{array}{l}337(1 \\
13- \\
588)\end{array}$ & $\begin{array}{l}0.5 \\
51\end{array}$ \\
\hline $\begin{array}{l}\text { rektum } 0.1 \mathrm{c} \\
\text { c1frc }\end{array}$ & $\begin{array}{l}1 \\
5\end{array}$ & $\begin{array}{l}501(1 \\
77- \\
760)\end{array}$ & 19 & $\begin{array}{l}502(36 \\
8-676)\end{array}$ & 19 & \begin{tabular}{|l}
$500(2$ \\
$94-$ \\
$822)$
\end{tabular} & $\begin{array}{l}0.6 \\
69\end{array}$ \\
\hline $\begin{array}{l}\text { mesane } 0.1 \\
\text { cc1frc }\end{array}$ & $\begin{array}{l}1 \\
5\end{array}$ & $\begin{array}{l}484(2 \\
42- \\
1096)\end{array}$ & 19 & $\begin{array}{l}521(42 \\
0-802)\end{array}$ & 19 & \begin{tabular}{|l}
$570(3$ \\
$81-$ \\
$1461)$
\end{tabular} & $\begin{array}{l}0.2 \\
79\end{array}$ \\
\hline $\begin{array}{l}\text { sigmoid0.1 } \\
\text { cc1frc }\end{array}$ & $\begin{array}{l}1 \\
5\end{array}$ & $\begin{array}{l}422(1 \\
22- \\
946)\end{array}$ & 20 & $\begin{array}{l}514(13 \\
9-816)\end{array}$ & 19 & $\begin{array}{l}476(1 \\
47- \\
800)\end{array}$ & $\begin{array}{l}0.8 \\
24\end{array}$ \\
\hline $\begin{array}{l}\text { Bladder } \\
\text { fullness } \\
\text { (cc) } 2 . f r c\end{array}$ & $\leq 9$ & & & -110 & $>1$ & & \\
\hline (cGy) & $\mathrm{n}$ & $\begin{array}{l}\text { Mean } \\
\text { (Min- } \\
\text { Max) } \\
\end{array}$ & $\mathrm{n}$ & $\begin{array}{l}\text { Mean } \\
\text { (Min- } \\
\text { Max) }\end{array}$ & $\mathrm{n}$ & $\begin{array}{l}\text { Mean } \\
\text { (Min- } \\
\text { Max) }\end{array}$ & $\mathrm{p}$ \\
\hline $\begin{array}{l}\text { rectum2cc } \\
2 \mathrm{frc}\end{array}$ & $\begin{array}{l}1 \\
7\end{array}$ & $\begin{array}{l}365(25 \\
2-483)\end{array}$ & 16 & $\begin{array}{l}369(9 \\
6-553)\end{array}$ & $\begin{array}{l}2 \\
2\end{array}$ & $\begin{array}{l}352(2 \\
03- \\
579)\end{array}$ & $\begin{array}{l}0.4 \\
38\end{array}$ \\
\hline $\begin{array}{l}\text { mesane2cc } \\
2 \text { frc }\end{array}$ & $\begin{array}{l}1 \\
7\end{array}$ & $\begin{array}{l}387(30 \\
7-519)\end{array}$ & 16 & $\begin{array}{l}390(3 \\
35- \\
573)\end{array}$ & $\begin{array}{l}2 \\
2\end{array}$ & $\begin{array}{l}460(3 \\
23- \\
643)\end{array}$ & $\begin{array}{l}0.0 \\
41\end{array}$ \\
\hline $\begin{array}{l}\text { sigmoid } 2 \\
\text { cc2frc }\end{array}$ & $\begin{array}{l}1 \\
7\end{array}$ & $\begin{array}{l}226(10 \\
6-559)\end{array}$ & 16 & $\begin{array}{l}346(1 \\
50- \\
557) \\
\end{array}$ & $\begin{array}{l}2 \\
2\end{array}$ & $\begin{array}{l}302(7 \\
3- \\
463) \\
\end{array}$ & $\begin{array}{l}0.2 \\
71\end{array}$ \\
\hline $\begin{array}{l}\text { rectum } 1 \mathrm{cc} \\
2 \mathrm{frc}\end{array}$ & $\begin{array}{l}1 \\
7\end{array}$ & $\begin{array}{l}419(27 \\
8-529)\end{array}$ & 16 & $\begin{array}{l}432(1 \\
49- \\
643) \\
\end{array}$ & $\begin{array}{l}2 \\
2\end{array}$ & $\begin{array}{l}387(2 \\
30- \\
640) \\
\end{array}$ & $\begin{array}{l}0.9 \\
68\end{array}$ \\
\hline $\begin{array}{l}\text { mesane } 1 \mathrm{cc} \\
2 \mathrm{frc}\end{array}$ & $\begin{array}{l}1 \\
7\end{array}$ & $\begin{array}{l}415(34 \\
0-527)\end{array}$ & 16 & $\begin{array}{l}430(3 \\
40- \\
628)\end{array}$ & $\begin{array}{l}2 \\
2\end{array}$ & $\begin{array}{l}485(2 \\
52- \\
713)\end{array}$ & $\begin{array}{l}0.2 \\
33\end{array}$ \\
\hline $\begin{array}{l}\text { sigmoid } \\
1 \mathrm{cc} 2 \mathrm{frc}\end{array}$ & $\begin{array}{l}1 \\
7\end{array}$ & $\begin{array}{l}265(10 \\
8-527)\end{array}$ & 16 & $\begin{array}{l}401(1 \\
72- \\
637)\end{array}$ & $\begin{array}{l}2 \\
2\end{array}$ & $\begin{array}{l}341(8 \\
1- \\
519)\end{array}$ & $\begin{array}{l}0.2 \\
22\end{array}$ \\
\hline $\begin{array}{l}\text { rektum0.1c } \\
\text { c } 2 \text { frc }\end{array}$ & $\begin{array}{l}1 \\
7\end{array}$ & $\begin{array}{l}496(34 \\
9-632)\end{array}$ & 16 & $\begin{array}{l}505(1 \\
74- \\
811) \\
\end{array}$ & $\begin{array}{l}2 \\
2\end{array}$ & $\begin{array}{l}464(2 \\
94- \\
800) \\
\end{array}$ & $\begin{array}{l}0.9 \\
89\end{array}$ \\
\hline mesane0.1 & 1 & $482(38$ & 16 & $567(4$ & 2 & $592(3$ & 0.1 \\
\hline
\end{tabular}

\begin{tabular}{|c|c|c|c|c|c|c|c|}
\hline $\mathrm{cc} 2 \mathrm{frc}$ & 7 & 8-814) & & $\begin{array}{l}10- \\
861)\end{array}$ & 2 & $\begin{array}{l}61- \\
1121) \\
\end{array}$ & 84 \\
\hline $\begin{array}{l}\text { sigmoid0.1 } \\
\text { cc } 2 \text { frc }\end{array}$ & $\begin{array}{l}1 \\
7\end{array}$ & $\begin{array}{l}380(12 \\
0-765)\end{array}$ & 16 & $\begin{array}{l}476(2 \\
05- \\
809)\end{array}$ & $\begin{array}{l}2 \\
2\end{array}$ & $\begin{array}{l}441(1 \\
00- \\
632)\end{array}$ & $\begin{array}{l}0.5 \\
08\end{array}$ \\
\hline $\begin{array}{l}\text { Bladder } \\
\text { fullness } \\
\text { (cc) 3.frc }\end{array}$ & \multicolumn{2}{|c|}{$\leq 90$} & \multicolumn{2}{|c|}{$90-110$} & & $>110$ & \\
\hline (cGy) & $\mathrm{n}$ & $\begin{array}{l}\text { Mean } \\
\text { (Min- } \\
\text { Max) }\end{array}$ & $\mathrm{n}$ & $\begin{array}{l}\text { Mean } \\
\text { (Min- } \\
\text { Max) } \\
\end{array}$ & $\mathrm{n}$ & $\begin{array}{l}\text { Mean } \\
\text { (Min- } \\
\text { Max) }\end{array}$ & $\mathrm{p}$ \\
\hline $\begin{array}{l}\text { rectum2cc } \\
3 \text { frc }\end{array}$ & $\begin{array}{l}1 \\
1\end{array}$ & $\begin{array}{l}343(13 \\
2-456)\end{array}$ & 17 & $\begin{array}{l}360(1 \\
02- \\
517) \\
\end{array}$ & $\begin{array}{l}2 \\
7\end{array}$ & $\begin{array}{l}386(4 \\
4- \\
589) \\
\end{array}$ & $\begin{array}{l}0.2 \\
93\end{array}$ \\
\hline $\begin{array}{l}\text { mesane } 2 \mathrm{cc} \\
3 \mathrm{frc}\end{array}$ & $\begin{array}{l}1 \\
1\end{array}$ & $\begin{array}{l}399(32 \\
3-589)\end{array}$ & 17 & $\begin{array}{l}435(3 \\
53- \\
569) \\
\end{array}$ & $\begin{array}{l}2 \\
7\end{array}$ & $\begin{array}{l}432(2 \\
21- \\
637)\end{array}$ & $\begin{array}{l}0.7 \\
36\end{array}$ \\
\hline $\begin{array}{l}\text { sigmoid2c } \\
\text { c } 3 \text { frc }\end{array}$ & $\begin{array}{l}1 \\
1\end{array}$ & $\begin{array}{l}189(12 \\
3-510)\end{array}$ & 17 & $\begin{array}{l}281(9 \\
1-551)\end{array}$ & $\begin{array}{l}2 \\
7\end{array}$ & $\begin{array}{l}291(5 \\
7- \\
693)\end{array}$ & $\begin{array}{l}0.2 \\
82\end{array}$ \\
\hline $\begin{array}{l}\text { rectum } 1 \mathrm{cc} \\
3 \mathrm{frc}\end{array}$ & $\begin{array}{l}1 \\
1\end{array}$ & $\begin{array}{l}401(15 \\
3-518)\end{array}$ & 17 & $\begin{array}{l}90- \\
561) \\
\end{array}$ & $\begin{array}{l}2 \\
7\end{array}$ & $\begin{array}{l}446(2 \\
81- \\
657) \\
\end{array}$ & $\begin{array}{l}0.0 \\
55\end{array}$ \\
\hline $\begin{array}{l}\text { mesane1cc } \\
3 \text { frc }\end{array}$ & $\begin{array}{l}1 \\
1\end{array}$ & & 17 & $\begin{array}{l}489(3 \\
86- \\
627) \\
\end{array}$ & $\begin{array}{l}2 \\
7\end{array}$ & $\begin{array}{l}500(3 \\
27- \\
856) \\
\end{array}$ & $\begin{array}{l}0.8 \\
01\end{array}$ \\
\hline $\begin{array}{l}\text { sigmoid } \\
1 \mathrm{cc} 3 \mathrm{frc}\end{array}$ & $\begin{array}{l}1 \\
1\end{array}$ & $\begin{array}{l}213(14 \\
3-582)\end{array}$ & 17 & $\begin{array}{l}333(1 \\
00- \\
545) \\
\end{array}$ & $\begin{array}{l}2 \\
7\end{array}$ & $\begin{array}{l}307(6 \\
4- \\
623) \\
\end{array}$ & $\begin{array}{l}0.3 \\
61\end{array}$ \\
\hline $\begin{array}{l}\text { rectum0.1c } \\
\text { c } 3 \text { frc }\end{array}$ & $\begin{array}{l}1 \\
1\end{array}$ & $\begin{array}{l}481(55- \\
665)\end{array}$ & 17 & $\begin{array}{l}461(2 \\
52- \\
644)\end{array}$ & $\begin{array}{l}2 \\
7\end{array}$ & $\begin{array}{l}537(3 \\
65- \\
876)\end{array}$ & $\begin{array}{l}0.0 \\
24\end{array}$ \\
\hline $\begin{array}{l}\text { mesane } 0.1 \\
\text { cc } 3 \text { frc }\end{array}$ & $\begin{array}{l}1 \\
1\end{array}$ & $\begin{array}{l}565(41 \\
5-917)\end{array}$ & 17 & $\begin{array}{l}577(4 \\
56- \\
837) \\
\end{array}$ & $\begin{array}{l}2 \\
7\end{array}$ & $\begin{array}{l}549(3 \\
80- \\
902) \\
\end{array}$ & $\begin{array}{l}0.5 \\
69\end{array}$ \\
\hline $\begin{array}{l}\text { sigmoid0.1 } \\
\text { cc 3frc }\end{array}$ & $\begin{array}{l}1 \\
1\end{array}$ & $\begin{array}{l}281(19 \\
5-812)\end{array}$ & 17 & $\begin{array}{l}410(1 \\
10- \\
752) \\
\end{array}$ & $\begin{array}{l}2 \\
7\end{array}$ & $\begin{array}{l}421(8 \\
2- \\
701) \\
\end{array}$ & $\begin{array}{l}0.3 \\
92\end{array}$ \\
\hline
\end{tabular}

$\mathrm{P}=<0,05$

When examined according to operation presence, the bladder spot dose was found to be statistically significant in operated patients $(\mathrm{p}=0.005)$ (table 4$)$. Although it was less effective on rectum doses when compared in terms of operation status, it was also observed that it increased organs at risk doses in all fractions $(\mathrm{p}<0.001)$ (Table 5). When compared in terms of the type of application, the bladder dose was significantly higher in cylinder applications compared to the other two applications $(\mathrm{p}=0.001) \quad$ (Table 6). Today, the Intracavitary Brachytherapy application is applied according to the bone structure with BT-planning in the first fraction and with conventional graphics in the following fractions in many centers. Anatomical changes of the target and organs at risk might change the applicator placement angle, which can change the target and organs at risk doses among fractions. When Adaptive Brachytherapy is applied with the guidance of imaging, the volume definition may be made with high accuracy by seeing changes in the targets and OARs by performing CT in each fraction $[6,7,8]$. 
Table 4: Comparison of OAR, Volume and Fraction Variables according to Operation Presence

\begin{tabular}{|c|c|c|c|c|c|c|c|}
\hline & \multicolumn{3}{|c|}{ Operation } & \multicolumn{3}{|c|}{ No Operation } & \\
\hline $\begin{array}{l}\text { OAR/Vol } \\
\text { ume/fr no } \\
\text { (cGy) }\end{array}$ & $\mathrm{N}$ & $\begin{array}{l}\text { Mea } \\
\mathrm{n} \pm \mathrm{S} \\
\mathrm{D}\end{array}$ & $\begin{array}{l}\text { Mean } \\
\text { (Min- } \\
\text { Max) }\end{array}$ & $\mathrm{N}$ & $\begin{array}{l}\text { Mea } \\
\mathrm{n} \pm \mathrm{S} \\
\mathrm{D}\end{array}$ & $\begin{array}{l}\text { Mean } \\
\text { (Min- } \\
\text { Max) }\end{array}$ & $\mathrm{p}$ \\
\hline $\begin{array}{l}\text { Rectum } \\
2 \mathrm{cc} 1 . \mathrm{frc}\end{array}$ & 3 & $\begin{array}{l}404 . \\
8 \pm 7 \\
7.8\end{array}$ & $\begin{array}{l}391.6( \\
235.2- \\
561.7)\end{array}$ & $\begin{array}{l}2 \\
5\end{array}$ & $\begin{array}{l}348 . \\
5 \pm 1 \\
17.9\end{array}$ & $\begin{array}{l}329.0( \\
72.0- \\
549.7)\end{array}$ & $\begin{array}{l}\mathbf{0 .} \\
\mathbf{0 3} \\
\mathbf{0} \\
\end{array}$ \\
\hline $\begin{array}{l}\text { Rectum } \\
\text { 2cc 2.frc }\end{array}$ & 3 & $\begin{array}{l}385 \\
7 \pm 6 \\
2.2 \\
\end{array}$ & $\begin{array}{l}385.2( \\
246.0- \\
524.0) \\
\end{array}$ & $\begin{array}{l}2 \\
5\end{array}$ & $\begin{array}{l}319 . \\
0 \pm 1 \\
20.6 \\
\end{array}$ & $\begin{array}{l}298 \\
96 . \\
579 \\
\end{array}$ & \begin{tabular}{|l|}
0. \\
01 \\
1 \\
\end{tabular} \\
\hline $\begin{array}{l}\text { Rectl } \\
2 \mathrm{cc} 3\end{array}$ & 3 & $\begin{array}{l}394 . \\
9 \pm 6 \\
3.8 \\
\end{array}$ & $\begin{array}{l}384.5( \\
258.7- \\
528.4) \\
\end{array}$ & $\begin{array}{l}2 \\
5\end{array}$ & $\begin{array}{l}332 . \\
1 \pm 1 \\
28.3 \\
\end{array}$ & $\begin{array}{l}365.0( \\
44.0- \\
589.0) \\
\end{array}$ & $\begin{array}{l}0 . \\
06 \\
2 \\
\end{array}$ \\
\hline $\begin{array}{l}\text { Rectum } \\
\text { 2cc 4.frc }\end{array}$ & 1 & $\begin{array}{l}408 . \\
7 \pm 6 \\
9.7 \\
\end{array}$ & $\begin{array}{l}397.0( \\
286.0- \\
521.2)\end{array}$ & $\begin{array}{l}1 \\
5\end{array}$ & $\begin{array}{l}311 . \\
0 \pm 9 \\
5.0 \\
\end{array}$ & $\begin{array}{l}314.0( \\
93.0- \\
447.4) \\
\end{array}$ & \begin{tabular}{|l|}
0. \\
01 \\
3 \\
\end{tabular} \\
\hline & 3 & $\begin{array}{l}395 \\
2 \pm 6 \\
6.1\end{array}$ & $\begin{array}{l}387.4( \\
246.0- \\
570.6)\end{array}$ & $\begin{array}{l}2 \\
5\end{array}$ & $\begin{array}{l}464 . \\
0 \pm 1 \\
04.1\end{array}$ & & $\begin{array}{l}0 . \\
00 \\
5\end{array}$ \\
\hline $\begin{array}{l}\text { Mesane } \\
\text { 2cc 2.frc }\end{array}$ & 0 & $\begin{array}{l}393 . \\
4 \pm 6 \\
1.5 \\
\end{array}$ & $\begin{array}{l}383.5( \\
307.0- \\
543.0) \\
\end{array}$ & $\begin{array}{l}2 \\
5\end{array}$ & $\begin{array}{l}458 . \\
0 \pm 8 \\
8.0\end{array}$ & & $\begin{array}{l}0 . \\
00 \\
7 \\
\end{array}$ \\
\hline $\begin{array}{l}\text { Mes } \\
2 \mathrm{cc}\end{array}$ & U & $\begin{array}{l}393 . \\
6 \pm 5 \\
6.6 \\
\end{array}$ & $\begin{array}{l}387.8( \\
298.0- \\
516.0) \\
\end{array}$ & $\begin{array}{l}2 \\
5\end{array}$ & $\begin{array}{l}468 . \\
2 \pm 8 \\
7.0 \\
\end{array}$ & $\begin{array}{l}479.0( \\
221.0- \\
637.1) \\
\end{array}$ & \begin{tabular}{|l|}
0. \\
00 \\
01 \\
\end{tabular} \\
\hline & & $\begin{array}{l}395 . \\
7 \pm 6 \\
8.0\end{array}$ & $\begin{array}{l}366.0( \\
305.0- \\
516.3)\end{array}$ & $\begin{array}{l}1 \\
5\end{array}$ & $\begin{array}{l}457 . \\
8 \pm 5 \\
1.9\end{array}$ & & $\begin{array}{l}0 . \\
04 \\
1\end{array}$ \\
\hline $\begin{array}{l}\text { Sigmoid } \\
2 \mathrm{cc} 1 . \mathrm{frc}\end{array}$ & $\begin{array}{l}3 \\
0\end{array}$ & $\begin{array}{l}241 . \\
7 \pm 1 \\
00.4\end{array}$ & $\begin{array}{l}246.7( \\
92.0- \\
449.0)\end{array}$ & $\begin{array}{l}2 \\
5\end{array}$ & $\begin{array}{l}364 . \\
8 \pm 1 \\
12.9\end{array}$ & $\begin{array}{l}369.0( \\
143.0- \\
582.0)\end{array}$ & $\begin{array}{l}0 . \\
00 \\
01\end{array}$ \\
\hline $\begin{array}{l}\text { Sigmoid } \\
2 \mathrm{cc} 2 \text {.frc }\end{array}$ & 0 & $\begin{array}{l}242 . \\
9 \pm 1 \\
17.1 \\
\end{array}$ & $\begin{array}{l}210.8( \\
73.3- \\
455.0)\end{array}$ & 2 & $\begin{array}{l}372 . \\
8 \pm 1 \\
12.5 \\
\end{array}$ & $\begin{array}{l}368.0( \\
143.0- \\
559.0)\end{array}$ & \begin{tabular}{|l|}
0. \\
00 \\
01 \\
\end{tabular} \\
\hline $\begin{array}{l}\text { Sigmoid } \\
2 \mathrm{cc} 3 . \mathrm{frc}\end{array}$ & $\begin{array}{l}3 \\
0\end{array}$ & $\begin{array}{l}215 . \\
5 \pm 1 \\
06.4\end{array}$ & $\begin{array}{l}204.1( \\
57.4- \\
44.0) \\
\end{array}$ & $\begin{array}{l}2 \\
5\end{array}$ & $\begin{array}{l}372 . \\
8 \pm 1 \\
39.9 \\
\end{array}$ & $\begin{array}{l}333.0( \\
174.3- \\
693.4)\end{array}$ & \begin{tabular}{|l|}
0. \\
00 \\
01 \\
\end{tabular} \\
\hline $2 \mathrm{cc} 4$ & 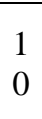 & $\begin{array}{l}178 . \\
7 \pm 7 \\
5.8\end{array}$ & $\begin{array}{l}202.0( \\
55.0- \\
277.3)\end{array}$ & 1 & $\begin{array}{l}400 . \\
9 \pm 1 \\
09.4\end{array}$ & $\begin{array}{l}393.0( \\
206.0- \\
581.0)\end{array}$ & $\begin{array}{l}0 . \\
00 \\
01\end{array}$ \\
\hline
\end{tabular}

$\mathrm{P}=<0,05$

Table 5: Evaluation of the Effect of the Operation Factor on Organ at Risk Volumes

\begin{tabular}{|l|c|c|c|}
\hline $\begin{array}{l}\text { Operati } \\
\text { on }\end{array}$ & Yes & No & \\
\hline rectum & Med (Min-Max) & Med (Min-Max) & $\mathrm{p}$ \\
\hline 1cc1frc & $\begin{array}{c}427.5(270.3- \\
610.9)\end{array}$ & $\begin{array}{c}381.1(147.0- \\
620.5)\end{array}$ & 0.071 \\
\hline r1cc2frc & $\begin{array}{c}422.5(278.0- \\
579.2)\end{array}$ & $\begin{array}{c}368.0(149.0- \\
643.0)\end{array}$ & $\mathbf{0 . 0 4 0}$ \\
\hline r1cc 3frc & $\begin{array}{c}437.3(290.6- \\
657.5)\end{array}$ & $\begin{array}{c}384.0(153.0- \\
620.3)\end{array}$ & $\mathbf{0 . 0 3 3}$ \\
\hline r1cc 4frc & $\begin{array}{c}440.0(279.0- \\
571.2)\end{array}$ & $\begin{array}{c}370.0(198.0- \\
555.5)\end{array}$ & 0.305 \\
\hline r1cc5frc & $\begin{array}{c}350.0(313.0- \\
416.0)\end{array}$ & - & \\
\hline
\end{tabular}

\begin{tabular}{|c|c|c|c|}
\hline $\begin{array}{l}\text { mesane1 } \\
\text { cc } 1 \text { frc }\end{array}$ & $\begin{array}{c}420.7(273.0- \\
614.0)\end{array}$ & $\begin{array}{c}493.5(301.0- \\
743.0)\end{array}$ & 0.010 \\
\hline $\begin{array}{l}\mathrm{m} 1 \mathrm{cc} \\
2 \mathrm{frc}\end{array}$ & $\begin{array}{c}406.5(252.0- \\
616.5)\end{array}$ & $\begin{array}{c}512.7(340.0- \\
712.6)\end{array}$ & 0.001 \\
\hline $\begin{array}{l}\text { m1cc } \\
3 \text { frc }\end{array}$ & $\begin{array}{c}436.5(327.0- \\
856.0)\end{array}$ & $\begin{array}{c}519.0(354.0- \\
693.4)\end{array}$ & $\begin{array}{c}0.000 \\
1\end{array}$ \\
\hline $\begin{array}{l}\text { m1cc } \\
4 \text { frc }\end{array}$ & $\begin{array}{c}428.0(331.0- \\
558.0)\end{array}$ & $\begin{array}{c}517.4(448.0- \\
583.0)\end{array}$ & 0.002 \\
\hline $\begin{array}{l}\mathrm{m} 1 \mathrm{cc} \\
5 \mathrm{frc}\end{array}$ & $\begin{array}{c}387.0(310.0- \\
391.0)\end{array}$ & - & \\
\hline $\begin{array}{l}\text { sigmoid } \\
1 \mathrm{cc} 1 \mathrm{frc}\end{array}$ & $\begin{array}{c}281.8(97.5- \\
553.0)\end{array}$ & $\begin{array}{c}427.0(154.5- \\
648.0)\end{array}$ & 0.001 \\
\hline s1cc 2 frc & $\begin{array}{c}247.5(80.7- \\
504.0)\end{array}$ & $\begin{array}{c}420.0(160.0- \\
637.4)\end{array}$ & 0.001 \\
\hline s $1 \mathrm{cc} 3 \mathrm{frc}$ & $\begin{array}{c}240.3(64.0- \\
504.0)\end{array}$ & $\begin{array}{c}365.0(199.0- \\
623.0)\end{array}$ & $\begin{array}{c}0.000 \\
1\end{array}$ \\
\hline
\end{tabular}

Table 6: Evaluation of the Effect of Applicator Type on Organs at Risk Volumes

\begin{tabular}{|c|c|c|c|c|c|}
\hline $\begin{array}{l}\text { Appli } \\
\text { cator } \\
\text { Type }\end{array}$ & Cylinder & $\begin{array}{l}\text { Tandem } \\
\text { ring }\end{array}$ & $\begin{array}{l}\text { Tandem } \\
\text { cylinder }\end{array}$ & & \\
\hline & $\begin{array}{l}\text { Med } \\
\text { (Min- } \\
\text { Max) }\end{array}$ & $\begin{array}{l}\text { Med } \\
\text { (Min- } \\
\text { Max) }\end{array}$ & $\begin{array}{l}\text { Med } \\
\text { (Min- } \\
\text { Max) }\end{array}$ & $\mathrm{p}$ & $\mathrm{p}^{*}$ \\
\hline $\begin{array}{l}\text { rectu } \\
\mathrm{m}\end{array}$ & $427.5(270$ & $385.0(147$ & $377.2(292$ & 0.1 & 0.0 \\
\hline $\begin{array}{l}1 \mathrm{cc} 1 \mathrm{f} \\
\mathrm{rc}\end{array}$ & $.3-610.9)$ & $.0-620.5)$ & $.0-602.3)$ & 95 & 75 \\
\hline $\begin{array}{l}\text { r1cc2 } \\
\text { frc }\end{array}$ & $\begin{array}{c}422.5(278 \\
.0-579.2)\end{array}$ & $\begin{array}{c}356.5(149 \\
.0-643.0)\end{array}$ & $\begin{array}{c}368.0(283 \\
.0-640.0)\end{array}$ & $\begin{array}{l}0.1 \\
08\end{array}$ & $\begin{array}{l}0.0 \\
36\end{array}$ \\
\hline r1cc & $\begin{array}{r}437.3(290 \\
6-657.5)\end{array}$ & $\begin{array}{r}392.0(153 \\
0-6203)\end{array}$ & $\begin{array}{r}333.0(231 \\
0-5180)\end{array}$ & $\begin{array}{l}0.1 \\
03\end{array}$ & $\begin{array}{l}0.0 \\
40\end{array}$ \\
\hline $\begin{array}{l}\text { r1cc } \\
4 \text { frc }\end{array}$ & $\begin{array}{c}440.0(279 \\
.0-571.2)\end{array}$ & $\begin{array}{c}370.0(198 \\
.0-555.5)\end{array}$ & $\begin{array}{c}.462 .5(370 \\
.0-555.0)\end{array}$ & $\begin{array}{l}0.3 \\
73\end{array}$ & $\begin{array}{l}0.2 \\
28\end{array}$ \\
\hline $\begin{array}{l}\text { r1cc5 } \\
\text { frc }\end{array}$ & $\begin{array}{c}350.0(313 \\
.0-416.0)\end{array}$ & - & - & & \\
\hline $\begin{array}{l}\text { mesa } \\
\text { ne1c } \\
\text { c } 1 \text { frc }\end{array}$ & $\begin{array}{c}420.7(273 \\
.0-614.0)\end{array}$ & $\begin{array}{c}483.0(301 \\
.0-743.0)\end{array}$ & $\begin{array}{c}510.0(432 \\
.7-612.6)\end{array}$ & $\begin{array}{l}0.0 \\
32\end{array}$ & $\begin{array}{c}0.0 \\
19\end{array}$ \\
\hline $\begin{array}{l}\mathrm{m} 1 \mathrm{cc} \\
2 \mathrm{frc}\end{array}$ & $\begin{array}{c}406.5(252 \\
.0-616.5)\end{array}$ & $\begin{array}{c}505.4(340 \\
.0-712.6)\end{array}$ & $\begin{array}{c}527.0(463 \\
.5-681.0)\end{array}$ & $\begin{array}{c}0.0 \\
04 \\
\end{array}$ & $\begin{array}{c}0.0 \\
05 \\
\end{array}$ \\
\hline $\begin{array}{l}\mathrm{m} 1 \mathrm{cc} \\
3 \mathrm{frc}\end{array}$ & $\begin{array}{c}436.5(327 \\
.0-856.0)\end{array}$ & $\begin{array}{c}527.0(354 \\
.0-693.4)\end{array}$ & $\begin{array}{c}516.0(467 \\
.6-676.0)\end{array}$ & $\begin{array}{c}0.0 \\
00 \\
1\end{array}$ & $\begin{array}{c}0.0 \\
00 \\
1\end{array}$ \\
\hline $\begin{array}{l}\text { m1cc } \\
4 \mathrm{frc}\end{array}$ & $\begin{array}{c}428.0(331 \\
.0-558.0)\end{array}$ & $\begin{array}{c}517.4(448 \\
.0-583.0)\end{array}$ & $\begin{array}{c}521.5(511 \\
.0-532.0)\end{array}$ & $\begin{array}{l}0.0 \\
12\end{array}$ & $\begin{array}{c}0.0 \\
04\end{array}$ \\
\hline $\begin{array}{l}\text { m1cc } \\
5 \text { frc }\end{array}$ & $\begin{array}{c}387.0(310 \\
.0-391.0)\end{array}$ & - & - & & \\
\hline $\begin{array}{l}\text { sigm } \\
\text { oid } \\
\text { 1cc1f } \\
\text { rc }\end{array}$ & $\begin{array}{c}281.8(97 . \\
5-553.0)\end{array}$ & $\begin{array}{c}420.5(154 \\
.5-648.0)\end{array}$ & $\begin{array}{c}461.0(243 \\
.0-466.8)\end{array}$ & $\begin{array}{c}0.0 \\
05\end{array}$ & $\begin{array}{c}0.0 \\
01\end{array}$ \\
\hline $\begin{array}{l}\mathrm{s} 1 \mathrm{cc} \\
2 \mathrm{frc}\end{array}$ & $\begin{array}{c}247.5(80 \\
7-504.0)\end{array}$ & $\begin{array}{c}422.5(237 \\
.4-637.4)\end{array}$ & $\begin{array}{c}237.4(160 \\
.0-527.0)\end{array}$ & $\begin{array}{c}0.0 \\
01\end{array}$ & $\begin{array}{c}\mathbf{0 . 0} \\
00 \\
1\end{array}$ \\
\hline $\begin{array}{l}\text { s1cc } \\
3 \text { frc }\end{array}$ & $\begin{array}{c}240.3(64 \\
0-504.0)\end{array}$ & $\begin{array}{c}378.5(239 \\
.0-623.0)\end{array}$ & $\begin{array}{c}338.3(199 \\
.0-416.0)\end{array}$ & $\begin{array}{c}\mathbf{0 . 0} \\
00 \\
1\end{array}$ & $\begin{array}{c}0.0 \\
00 \\
1\end{array}$ \\
\hline
\end{tabular}

$\mathrm{P}=<0.05$ 


\section{Discussion}

There are many studies in the literature examining the effect of bladder volume on OAR doses [9, 10, 11]. Studies conducted with the standard bladder filling protocol in each application reported dosimetric changes stemming from the differences in organ volumes [11, 12]. In the study conducted by Jamema et al. in which they examined the OAR dose and volume changes, they found interfractional increases only in sigmoid doses, and associated this with the treatment applied by emptying the bladder in the same way in each fraction resulting in constant bladder and rectum volumes, due to the sigmoid being a more mobile organ [13]. In the present study, bladder fullness rates did not have any significant effects on OAR doses. This result can be explained with good bowel preparation before each application and standard bladder filling protocol $(20 \mathrm{cc}$ contoured SF with probe). Because the distribution of bladder fullness values were examined, the median residue urine amounts measured in planning BT were similar to each other (Table 2).

GEC ESTOAR (Group European de CurietherapieEuropean Society for Therapeutic Radiology and Oncology) and ABS recommended the dose of $2 \mathrm{cc}$ in organs at risk as the hot spot $\left(\mathrm{D}_{\max }\right)$, and $1 \mathrm{cc}$ and $0.1 \mathrm{cc}$ doses as spot doses [14, 15]. There are many studies evaluating the $\mathrm{D}_{2 \mathrm{cc}}, \mathrm{D}_{1 \mathrm{cc}}$ and $\mathrm{D}_{0.1 \mathrm{cc}}$ doses of organs at risk which show that bladder fullness affects rectum and sigmoid doses $[9,16,17,18,19]$. In our study, the dose of $2 \mathrm{cc}$ was used as $D_{\max }$, and bladder fullness was not found to be effective on OAR $D_{2 c c}$ values. When the interfractional changes of OAR, $\mathrm{D}_{2} \mathrm{cc}, \mathrm{D}_{1 \mathrm{cc}}$ and $\mathrm{D}_{0.1 \mathrm{cc}}$ were examined, there were significant differences only in sigmoid $\mathrm{D}_{1 \mathrm{cc}}$ and $\mathrm{D}_{0.1 \mathrm{cc}} 1^{\text {st }}$ and $3^{\text {rd }}$ fractions $(\mathrm{p}=0.004)$.

During planning, there may be anatomical variations in the bladder position, and some studies showed that there were anatomical changes in the bladder after hysterectomy [20]. When the effect of the applicator type and operation factors on OAR $\mathrm{D}_{2 \mathrm{cc}}, \mathrm{D}_{1 \mathrm{cc}}, \mathrm{D}_{0.1 \mathrm{cc}}$ were examined, although rectum doses were less affected, all dose values were found to be significantly higher in cylinder applications compared to tandem + ring applications because the choice of applicators in operated patients would be cylindrical. The dose distribution stemming from the shape of the applicators and the treatment of the vagina that is neighboring the bladder may have caused the OAR dose increase [12].

When the results of the studies were evaluated together, it can be seen that the bladder dose was higher in patients for whom cylinders were used, and the changes in the anatomical placements of organs at risk in operated patients contribute to this dose increase $[14,18$, $19,20]$.
In the present study, various factors that affected the $\mathrm{D}_{2}$ cc, $\mathrm{D}_{1 \mathrm{cc}}, \mathrm{D}_{0.1 \mathrm{cc}}$ values of the bladder, sigmoid and rectum. The operation status and the applicator type of the patients affected OAR doses, and bladder fullness had no statistically significant effect on organs at risk. It was also observed that although the presence of an operation was less effective on rectum doses, it increased organs at risk doses in all fractions (Table 5).

Similarly, when OAR doses were evaluated in terms of applicator shape, since the selection of applicator in operated patients would be cylindrical, OAR doses were statistically higher in cylinder applications than in tandem + ring applications however, rectum doses were less affected (Table 6).

The limitations of the present study were that although patients were taken into the treatment with nutritional advice and standard enema applications, rectum fullness could have a wide-range among the fractions. Thus, a more standard treatment approach may be possible with methods with which rectum fullness can be monitored objectively. Another limitation of our study was that the patient population was relatively low. Further studies with wider number of patients are needed.

\section{Conclusion}

3D Adaptive Brachytherapy (3D-ABRT) ensures that the volumes are determined in the most accurate way by observing the changes in target tissue and surrounding tissues in each application. In this way, it is possible to apply high doses directly to the target volume without increasing the morbidity risk in organs at risk. Furthermore, treatment accuracy can be increased with routine preparations before the treatment and by imaging in each fraction.

\section{References}

1. World Health Organization. Global Health Observatory. Geneva: World Health Organization; 2018. who.int/gho/database/en/. Accessed June 21, 2018.

2. Kizilkaya HO, Kutluhan Dogan A, Dag Z et all, A Comparison of Magnetic Resonance and Computed Tomography Imaging Based Target-Volume Definition and Interfraction Variations of Treatment Planning Parameters (D90 HR-CTV, D2cc for OARs) During Image Guided Adaptive Brachytherapy for Cervical Cancer. UHOD 2019; 29(4): 227-237.

3. Manea E, Escande A, Bockel S et al. Risk of Late Urinary Complications Following Image Guided Adaptive Brachytherapy for Locally Advanced Cervical Cancer: Refining Bladder Dose-Volume Parameters. Int J Radiat Oncol Biol Phys 2018 Jun 1;101(2):411-420

4. Westerveld H, Nesvacil N, Fokdal $L$ et al, Definitive radiotherapy with image-guided adaptive brachytherapy for primary vaginal cancer, Lancet Oncol. 2020 Mar;21(3):e157-e167. 
5. Tanderup K, Georg D, Pötter R, et al. Adaptive management of cervical cancer radiotherapy. Semin Radiat Oncol 2010:121-9.

6. Viswanathan AN, Thomadsen B American Brachytherapy Society Cervical Cancer Recommendations Committee, American Brachytherapy Society. American Brachytherapy Society consensus guidelines for locally advanced carcinoma of the cervix. Part I: General principles. Brachytherapy. 2012;11:33-46.

7. Dankulchai P, Petsuksiri J, Chansilpa Y, et al. Image-guided high-dose-rate brachytherapy in inoperable endometrial cancer. $\mathrm{Br} \quad \mathrm{J}$ Radiol. 2014;87:20140018.

8. Van den Bos W, Beriwal S, Velema L, et al. Image guided adaptive brachytherapy for cervical cancer: Dose contribution to involved pelvic nodes in two cancer centers. J Contemp Brachytherapy. 2014;6:21-7.

9. Rangarajan R. Interfraction Variations in Organ Filling and Their Impact on Dosimetry in CT Image Based HDR Intracavitary Brachytherapy. J Med Phys. 2018;43(1):23- 27

10. Patel RB, Gururajachar JM, Revathy T, Implications of bladder volume on target coverage and dose to critical structures during high dose rate intracavitary brachytherapy for cervical cancer: A dosimetric study. J Cancer Res Ther. Oct-Dec 2019;15(6):1207-1211.

11. Kim R. Y, M.D, Shen S, Lin HY, et al. Effects of bladder distension on organs at risk in 3D imagebased planning of intracavitary brachytherapy for cervical cancer. Int $\mathbf{J}$ Radiat Oncol Biol Phys. 2010;76:485-9

12. Adli M, Garipagaoglu M, Kocak Z. Effect of bladder distention on bladder base dose in gynaecological intracavitary high dose rate brachytherapy. Br J Radiol. 2009;82:243-8.

13. Jamema S.V, Mahantshetty U, Tanderup K, et all. Inter-application variation of dose and spatial location of $\mathrm{D} 2 \mathrm{~cm} 3$ volumes of OARs during MR image based cervix brachytherapy 2013; Radiotherapy and Oncology 107: 58-62

14. The GEC-ESTOAR Handbook of Brachytherapy. http://www.estro-education.org/ publications/ Documents/ GEC-ESTOARHandbookof Brachytherapy.html

15. Viswanathan AN, Erickson BA. Three-dimensional imaging in gynecologic brachytherapy: a survey of the American Brachytherapy Society. Int J Radiat Oncol Biol Phys. 2010;76:104-9.

16. Davidson MT, Yuen J, D'Souza DP, et al. Imageguided cervix high-dose-rate brachytherapy treatment planning: Does custom computed tomography planning for each insertion provide better conformal avoidance of organs at risk? Brachytherapy. 2008;7:37-42.

17. Kirisits C, Lang S, Dimopoulos J, et al. Uncertainties when using only one MRI-based treatment plan for subsequent high-dose-rate tandem and ring applications in brachytherapy of cervix cancer. Radiother Oncol. 2006;81:269-75.
18. Cengiz M, Gurdalli S, Selek U, et al. Effect of bladder distensionn on dose distribution of intracavitary brachytherapy for cervical cancer: Three-dimensional computed tomography plan evaluation. Int $\mathrm{J}$ Radiat Oncol Biol Phys 2008;70:464-468.

19. Sun LM, Huang HY, Huand EY, et al. A prospective study to assess the bladder distention effects on dosimetry in intracavitary brachytherapy of cervical cancer via computer tomographyassisted techniques. Radiother Oncol 2005;77:7782.

20. Jhingran A, Salehpour M, Sam M et al., Vaginal motion and bladder and rectal volumes during pelvic intensity-modulated radiation therapy after hysterectomy, Int J Radiat Oncol Biol Phys. 2012 Jan 1;82(1):256-62. 